\title{
Alain Mabanckou, Verre Cassé
}

\section{Alessandro Corio}

\section{OpenEdition}

\section{Journals}

\section{Edizione digitale}

URL: https://journals.openedition.org/studifrancesi/46369

DOI: 10.4000/studifrancesi.46369

ISSN: 2421-5856

\section{Editore}

Rosenberg \& Sellier

\section{Edizione cartacea}

Data di pubblicazione: 1 octobre 2007

Paginazione: 484-485

ISSN: 0039-2944

\section{Notizia bibliografica digitale}

Alessandro Corio, «Alain Mabanckou, Verre Cassé», Studi Francesi [Online], 152 (LI | II) | 2007, online dal 30 novembre 2015, consultato il 24 novembre 2021. URL: http://journals.openedition.org/ studifrancesi/46369; DOI: https://doi.org/10.4000/studifrancesi.46369

Questo documento è stato generato automaticamente il 24 novembre 2021.

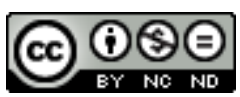

Studi Francesi è distribuita con Licenza Creative Commons Attribuzione - Non commerciale - Non opere derivate 4.0 Internazionale. 


\title{
Alain Mabanckou, Verre Cassé
}

\author{
Alessandro Corio
}

\section{NOTIZIA}

ALAin MABAnckou, Verre Cassé, Paris, Seuil, 2005, pp. 204.

1 «[...] c'est vraiment le désordre dans ce cahier, y a pas des points, il y a que des virgules et des virgules [...]».

2 Protagonista di questo bellissimo romanzo di Alain Mabanckou, vincitore di numerosi premi letterari, in particolare del Renaudot nel 2005, è la parola, o meglio, il suo scorrere apparentemente illimitato nella vertigine della scrittura. Dalla prima all'ultima pagina nemmeno un punto, nemmeno una pausa sintattica forte a spezzare questa continuità di parola e di racconto che, però, non è mai soffocante né invadente e, come un fiume che scorre lascia immaginare il suo letto profondo e cupo, lo scorrere della parola scritta è in costante presenza del fondo di silenzio cui, prima o poi, dovrà fare ritorno. Un atto d'amore per la letteratura, certo, quello che Mabanckou realizza affidando la voce narrante a Verre Cassé, un assiduo ed esuberante frequentatore di un locale, ironicamente denominato Le Crédit a voyagé (un'eco céliniana vi aleggia, uno dei numerosissimi rimandi intertestuali e citazioni disseminate nel testo), luogo di incontro di personaggi, di corpi, di tracce sporche di storie, di sofferenze grottesche e deliranti, ma profondamente umane: «disons que le patron du bar Le Crédit a voyagé m'a remis un cahier que je dois remplir, et il croit dur comme fer que moi, Verre Cassé, je peux pondre un livre parce que, en plaisantant, je lui avais raconté un jour l'histoire d'un écrivain célèbre qui buvait comme une éponge, un écrivain qu'on allait même ramasser dans la rue quand il était ivre [...]» (p. 11).

3 L'incipit del romanzo ci presenta immediatamente i due grandi temi che attraversano il suo intreccio: la scrittura e il bere, e subito dopo ne introduce un terzo, fondamentale, la memoria: «[...] j'ai voulu savoir pourquoi il tenait tant à ce cahier, il a répondu qu'il ne voulait pas que Le Crédit a voyagé disparaisse un jour comme ça, il a ajouté que les gens de ce pays n'avaient pas le sens de la conservation de la mémoire [...]» (p. 11). C'è una 
freschezza e un'energia nella scrittura di Mabanckou, che gli permette di mantenersi alla larga dai manierismi formali e soprattutto dai cliché esausti del discorso etnico, tradizionalista e pauperista sull'Africa, per immergersi nella rappresentazione della corporeità della vita, delle sue illusioni e disillusioni, dei suoi naufragi irreparabili di cui la parola, attraversando i più svariati registri, dal comico al grottesco, sino al registro più intimo della confessione personale, non vuole proporsi come salvezza, come "escatologia laica", ma come testimonianza e rappresentazione della traccia che anche le più meschine esistenze lasciano nei luoghi e nelle persone che (le) attraversano. Un bar quindi, quale luogo migliore per questa scrittura? Certo, Verre Cassé non è un osservatore imparziale, che, taccuino alla mano in veste di etnografo, osserva e descrive ciò che accade di fronte ai suoi occhi. No, al contrario, la sua esistenza è parte inscindibile di questo intreccio, costantemente minacciata dalla deriva e accompagnata da una scrittura che inizialmente appare, nella finzione, ben poco convinta e senz'altro non investita da nessuna missione o alto valore etico, ma che trova nel suo farsi il suo stesso senso, minacciata da una fine e da un abbandono sempre prossimi, ma costantemente differiti. Il punto, sorta di fantasma aleggiante dietro al racconto, non giungerà mai, mentre una compassione nascente che si infiltra lentamente nella scrittura di Verre Cassé lo spingerà, piuttosto, a un abbraccio con lo scorrere minaccioso di un fiume che, una volta accettato e amato, si rivelerà come il custode dell'amore di una madre.

Una volta accettata anche la sfida della scrittura, il nostro improvvisato narratore infila una serie di racconti esorbitanti, che vanno dal comico iperbolico e grottesco, costruendo una satira senza esclusione di colpi del linguaggio e della propaganda del potere, a episodi e personaggi raccontati sulle corde della derisione e dell'osceno, inscrivendosi così, a pieno titolo, in una tradizione del romanzo africano, evidenziata anche da Jacques Chevrier, che potrebbe eleggere come suoi "padri fondatori", se quest'atto ideale non fosse in assoluta divergenza con la sua linfa più anarchica $\mathrm{e}$ sovversiva, autori quali François Rabelais e Sony Labou Tansi. L'intero romanzo è pertanto intessuto di una miriade di riferimenti intertestuali in un vero e proprio pastiche di titoli di romanzi africani e mondiali, con un'abilità che non rallenta mai il ritmo eccezionale della narrazione e non appare come banale sfoggio di cultura, ma, come in un'ideale e babelica biblioteca vivente, riesce a fagocitare e "cannibalizzare" nel corpo stesso della sua scrittura i suoi riferimenti, con un meccanismo di appropriazione e di omaggio che non risulta meramente formale, e per di più divertendo il lettore in un gioco di rimandi, di riconoscimenti e di clins d'œil. Eccone un passaggio significativo: «...je me souviendrai toujours de ma première traversée d'un pays d'Afrique, c'était la Guinée, j'étais l'enfant noir (Camara Laye), j'étais fasciné par le labeur des forgerons, j'étais intrigué par la reptation d'un serpent mystique qui avalait un roseau que je croyais tenir réellement entre les mains, et très vite je retournais au pays natal (Césaire), je goûtais aux fruits si doux de l'arbre à pain (Tchicaya U tam'si), j'habitais dans une chambre de l'hôtel la vie et demie (Labou Tansi), qui n'existe plus de nos jours et où, chaque soir, entre jazz et vin de palme (Dongala), mon père aurait exulté de joie, et je me réchauffais au feu des origines (Dongala)...» (p. 171, nostri i corsivi e le indicazioni fra parentesi).

5 La sua scrittura resta pertanto semplice, a tratti dalle movenze infantili, trascinandoci in un ritmo che, pur mantenendosi su toni comici, riesce abilmente a mescolarvi il sublime del ricordo della madre e dell'infanzia. Il romanzo appare così diviso in due parti. Nella prima si succedono e si accavallano i ritratti e le storie di personaggi "hauts 
en couleur", sin dalla sfida iniziale tra le istituzioni sconvolte dalla nascita di questo bar, per cui ministri e presidenti si sfidano a colpi di slogan e di formule retoriche che, dal "j'accuse" iniziale, si concludono con un "je vous ai compris", a cui seguono una serie di personaggi segnati dal comune denominatore di una vita abortita che eccede ogni norma, di una "vie et demie", derelitti di una società in disfacimento, dall'“Homme aux Pampers", ingiustamente accusato ed orribilmente mutilato dall'esperienza della prigione, all'imprimeur, "l'homme qui a fait la France", implacabile satira dell'illusione perduta della salvezza nella "madrepatria", sino alla memorabile, epica e davvero rabelaisiana, sfida tra Robinette e Casimir le Géographe, vero e proprio trionfo del basso corporale e della carnevalizzazione del racconto.

6 Ma, afferma a un certo punto Verre Cassé, «il est trop facile de parler des autres et de ne pas parler de soi-même», e così, nella seconda parte del racconto, assistiamo a un vero e proprio "glissement vers l'écriture de soi", e il narratore, quasi sommerso dall'invadenza dei suoi personaggi che vogliono farsi raccontare, si concentra sulla propria storia, riaprendo delle piaghe e delle sofferenze che lasciano il segno nella scrittura stessa, spingendolo a più riprese a un gesto di rifiuto e di abbandono. Per quanto all'apparenza leggero e di facile e divertente, nonché trascinante in certi passaggi, lettura, il romanzo di Mabanckou è estremamente articolato e stratificato e meriterebbe un'analisi ben più estesa e approfondita dei temi che riesce a intrecciare, accompagnando una riflessione mai scontata sull'esistenza a quella sul senso dello scrivere e della letteratura. Egli condanna a più riprese coloro che non fanno altro che "filosofare senza vivere", elogiando così una scrittura che sappia "sporcarsi le mani" con la sostanza più viscerale, talora repulsiva, della vita. Ma il suo è un atto di amore anche nei confronti di una lingua, la lingua francese, che «n'est pas un long fleuve tranquille, [mais] c'est plutôt un fleuve à détourner» (p. 187). Un vero e proprio "corpo a corpo" con i dispositivi linguistici che, come afferma lo stesso protagonista, un tempo insegnante in una scuola da cui venne radiato per "inadeguatezza pedagogica", non può che realizzarsi in un atto di intima appropriazione e in una scoperta di sé e della propria umiltà, che è anche profonda iniziazione al vivere, oltre che un manifesto letterario: «[...] et j'ai aussi dit à L'Escargot entêté que si j'étais écrivain je demanderais à Dieu de me couvrir d'humilité, de me donner la force de relativiser ce que j'écris par rapport à ce que les géants de ce monde ont couché sur le papier [... ] ce n'est qu'à ce prix que j'écrirais des choses qui ressemblent à la vie, mais je les dirais avec des mots à moi, des mots tordus, des mots décousus, des mots sans queue ni tête [...]» (p. 198). Così, l'abbandono finale della scrittura e della vita per rigettarsi tra le braccia del fiume non ci appare come un'epica rinuncia al proprio sé, ma appunto come un bicchiere ormai a pezzi, ebbro del succo di vita che ha contenuto e determinato a ricongiungersi con "la madre", lasciando soltanto la traccia di parole scritte maldestramente. 